\title{
Sialólito gigante em glândula submandibular: relato de caso
}

\section{Giant sialolite in the submandibular gland: case report}

Sialolita gigante en la glándula submandibular: reporte de caso

Mateus Stenio Melo dos Santos

ORCID: https://orcid.org/0000-0003-1916-0656 Universidade Mário Pontes Jucá, Brasil E-mail: mateusmelos20@ hotmail.com

Laynne de Jesus Melo

ORCID: https://orcid.org/0000-0002-4140-3258 Universidade Mário Pontes Jucá, Brasil E-mail: laynnej@hotmail.com

Lucas Mariz de Menezes Torres ORCID: https://orcid.org/0000-0001-7543-7715 Centro Universitário Tiradentes, Brasil E-mail: 1marizdemenezes@gmail.com

Marielly dos Anjos Ferreira da Silva ORCID: https://orcid.org/0000-0001-8599-6385 Centro Universitário Tiradentes, Brasil E-mail: anjosmarielly@gmail.com

Davi Luiz Lage Cortez

ORCID: https://orcid.org/0000-0003-4517-5341 Centro Universitário Tiradentes, Brasil E-mail: davillagecortez@gmail.com

Darllon Manuel Borges Souza

ORCID: https://orcid.org/0000-0002-9858-6921 Centro Universitário Tiradentes, Brasil

E-mail: darllonmanuelsouzada@ hotmail.com

Kayo Costa Alves

ORCID: https://orcid.org/0000-0002-7788-820X

Hospital Policlin, Brasil

Clínica Prof. Antenor Araújo, Brasil

E-mail: kayocostaalves@gmail.com

José Ricardo Mikami

ORCID: https://orcid.org/0000-0002-2300-5117

Universidade de São Paulo, Brasil

E-mail: ricardomikami@hotmail.com

Ricardo José de Holanda Vasconcellos

ORCID: https://orcid.org/0000-0002-7934-5743

Universidade de Pernambuco, Brasil

E-mail: ricardo.holanda@upe.br

Marcus Antônio Brêda Júnior

ORCID: https://orcid.org/0000-0001-6515-472X

Universidade de São Paulo, Brasil

E-mail: marcusbredajr@gmail.com

\begin{abstract}
Resumo
Sialólito é uma patologia comum das glândulas salivares, principalmente as maiores, sendo a glândula submandibular mais acometida em 80 a $90 \%$ dos casos. Essa condição causa obstrução do ducto glandular, gerando acúmulo de saliva, inflamação, infecção, causando dor e desconforto ao paciente. Cálculos menores não excedem $10 \mathrm{~mm}$, mas maiores que $15 \mathrm{~mm}$ são considerados gigantes. A etiopatogenia pode estar ligada à deposição de cálcio em uma matriz de descamação celular, corpo estranho, muco, dentro de um ducto. Este artigo tem como objetivo relatar o diagnóstico e tratamento de um caso incomum quanto aos sintomas e tamanho de um sialólito na glândula submandibular esquerda.
\end{abstract}

Palavras-chave: Glândula submandibular; Sialolitíase; Cálculos de glândulas salivares.

\section{Abstract}

Sialolith is a common pathology of the salivary glands, especially the largest, with the most affected submandibular gland in 80 to $90 \%$ of cases. This condition causes obstruction of the gland duct, generating accumulation of saliva, inflammation, infection, causing pain and discomfort to the patient. Smaller calculations do not exceed $10 \mathrm{~mm}$, but larger than $15 \mathrm{~mm}$ are considered giants. The etiopathogenesis may be linked to the deposition of calcium in a matrix 
of cellular desquamation, foreign body, mucus, inside a duct. This article aims to report the diagnosis and treatment of an unusual case regarding the symptoms and size of a sialolith in the left submandibular gland.

Keywords: Submandibular gland; Sialolithiasis; Salivary gland calculi.

\section{Resumen}

El sialolito es una patología común de las glándulas salivales, especialmente las más grandes, con la glándula submandibular más afectada en el 80 al 90\% de los casos. Esta condición provoca la obstrucción del conducto de la glándula, generando acumulación de saliva, inflamación, infección, provocando dolor y malestar al paciente. Los cálculos más pequeños no superan los $10 \mathrm{~mm}$, pero los mayores de $15 \mathrm{~mm}$ se consideran gigantes. La etiopatogenia puede estar relacionada con la deposición de calcio en una matriz de descamación celular, cuerpo extraño, moco, dentro de un conducto. Este artículo tiene como objetivo reportar el diagnóstico y tratamiento de un caso inusual relacionado con la sintomatología y el tamaño de un sialolito en la glándula submandibular izquierda.

Palabras clave: Glándula submandibular; Sialolitiasis; Cálculos de glándulas salivales.

\section{Introdução}

A sialolitíase é uma patologia caracterizada pela formação de uma massa calcificada no interior das glândulas ou ductos salivares, causando obstrução no fluxo salivar, tal massa é denominada sialólito (Fialho et al., 2018; Alves et al., 2014 \& Goes et al., 2013). A glândula submandibular é acometida entre 80 a 90\% dos casos (Leite et al., 2018; Silva et al., 2018; Azenha et al., 2013 \& Manzi et al., 2010). Quanto à etiopatogenia, acredita que advenham da deposição de cálcio numa matriz no interior do ducto, e que essa matriz pode conter restos celulares, descamação de epitélio do ducto, bactérias, muco espesso ou corpo estranho, sem haver relação quanto às desordens metabólicas do cálcio e do fósforo para possível ocorrência dos cálculos (Neville et al., 2009 \& Oliveira et al., 2019).

Diagnóstico para sialolitíase necessita-se de anamnese minuciosa, exame extra e intraoral, buscando possível aumento de volume ou inflamação nas regiões de maior acometimento, como no assoalho de boca, contudo a palpação é necessária (Kignel et al., 2013 Dall'Magro et al., 2014 \& Pretto et al., 2007). Além disso, exames de imagem intra e extrabucal são essenciais, dentre os quais a tomografia computadorizada de feixe cônico (TCFC) é a melhor escolha, por proporcionar visualização tridimensional, possibilitando perfeita execução do planejamento (Kraaij et al., 2014 \& Lima et al., 2013).

$\mathrm{O}$ tratamento varia, de técnica conservadora à invasiva, sendo o tamanho do cálculo, o grau de obliteração do ducto e a localização, pontos chaves para escolha terapêutica. Geralmente, essas massas calcificadas são menores que $10 \mathrm{~mm}$ de diâmetro, já quando ultrapassam $15 \mathrm{~mm}$ são consideradas gigantes (Landgraf et al., 2006). Cálculos menores podem ser tratados com dieta ácida, massagem e estimulação de secreção salivar, utilização de sialogogos, enquanto os maiores cirurgicamente (Kawata et al., 2006; Cardoso \& Hoston 2012).

No pós-cirúrgico, existem algumas complicações que o paciente pode apresentar. Geralmente, estas podem ser graves quando realizam acesso extraoral, sendo danificação do nervo facial ou lingual a mais severa. A cicatriz facial, diminuição da excreção salivar são outros problemas associados e relatados na literatura (Araújo et al., 2011). O objetivo desse artigo é relatar o diagnóstico e tratamento de um caso incomum quanto ao sintoma e tamanho de sialólito em glândula submandibular.

\section{Metodologia}

Este artigo retrata um caso clínico, descritivo e qualitativo, no qual seguiu as diretrizes da técnica de Pereira et al., (2018). Para melhor compreensão e condução do referente caso foi realizada a anamnese minuciosa que associada aos exames de imagem resultou em informações que contribuíram para o planejamento e tratamento. Não sendo necessário o envolvimento do comitê de ética, visto que os princípios éticos foram seguidos e respeitados, através do termo de consentimento livre e esclarecido. 


\section{Caso Clínico}

Paciente compareceu ao ambulatório com queixa álgica e discreto aumento de volume endurecido em região submandibular esquerda há mais ou menos 3 anos, só buscando tratamento neste momento. Durante anamnese nega qualquer doença de base ou alergia medicamentosa. Ao exame físico (palpação) observa-se lesão endurecida, com pouca mobilidade na região referente à glândula submandibular esquerda, ausência de sinais flogísticos, adequada abertura bucal e sem assimetrias. Solicitada radiografia panorâmica na qual foi constatada presença de lesão circunscrita de mais ou menos $3 \mathrm{~cm}$ de diâmetro, radiopaca na região dos molares lado esquerdo (Figura 1).

Figura 1: Radiografia panorâmica pré-operatória - lesão radiopaca em região de molares inferiores do lado esquerdo.

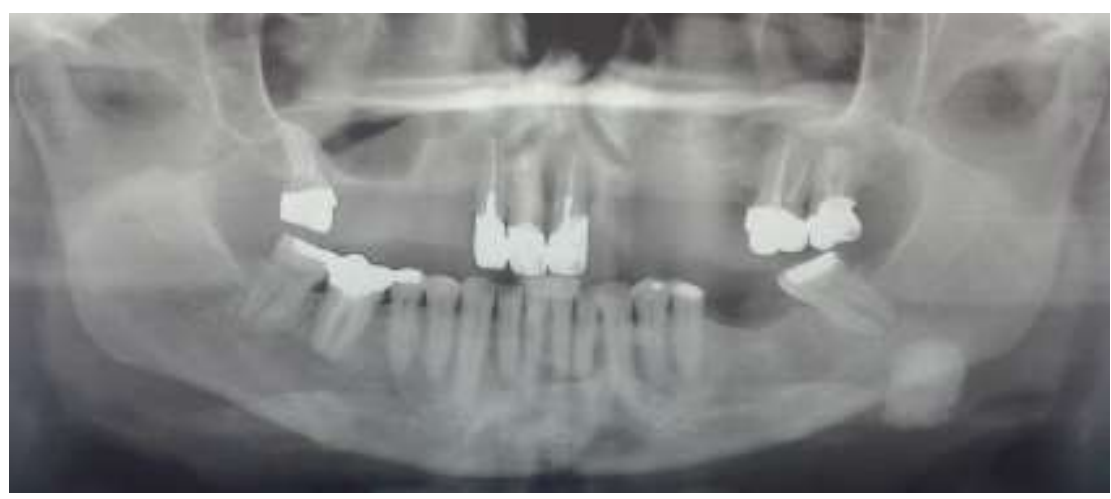

Fonte: Autores.

Em decorrência do tamanho foi sugerida a realização de tomografia computadorizada para uma precisa localização da lesão sugestiva de sialólito gigante. Nos cortes axial e coronal nota-se a presença de sialólito gigante na face interna mandibular, na região da glândula submandibular esquerda (Figura 2 (A e B)).

Figura 2: A: TC corte coronal pré-operatória, evidenciando extensa lesão radiopaca em região submandibular esquerda.

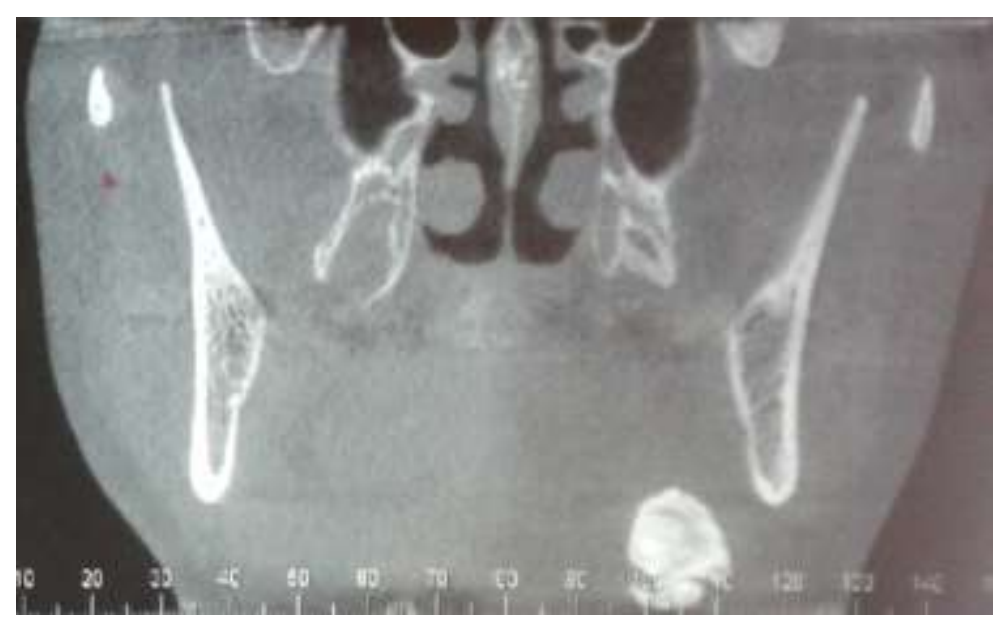

Fonte: Autores. 
Figura 2: B: TCFC corte axial pré-operatória, evidenciando extensa lesão radiopaca em região submandibular esquerda.

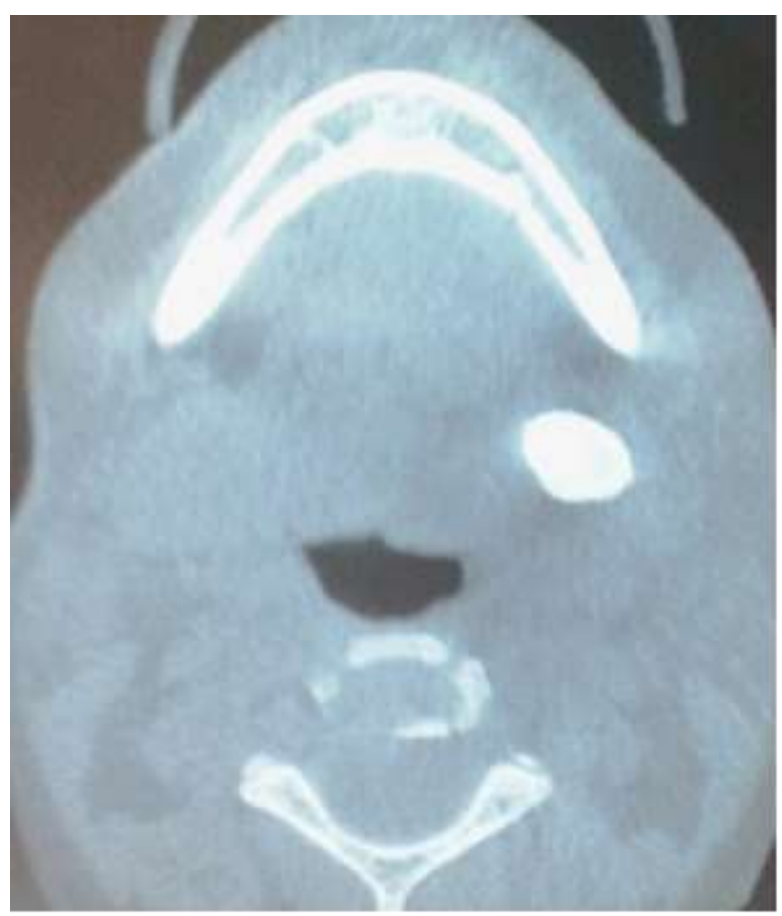

Fonte: Autores.

Cirurgia escolhida foi exérese da lesão. Paciente submetida à entubação nasotraqueal em decúbito dorsal horizontal, sob anestesia geral. Realizada antissepsia intraoral e extraoral com clorexidina 2,0\% degermante, aposição dos campos estéreis, inserção do tampão orofaríngeo. Infiltração em pele de adrenalina 1:1.000.00 (10:1) com intuito de realizar hemostasia. Incisão submandibular em pele $1,5 \mathrm{~cm}$ abaixo da borda mandibular, divulsão subcutânea e incisão e divulsão do músculo platisma e sistema músculo aponeurótico superficial em direção à glândula. Identificado o ramo marginal mandibular, artéria e veia facial, protegido e afastado do campo operatório. Acesso à glândula submandibular com aspecto aumentado de tamanho e com consistência firme a palpação. Incisão na parede glandular para remoção do sialólito na parte interna e necessidade de exérese total da glândula (Figura 3 (A, B e C)). Sutura interna com pontos simples realizada com fio Vicryl 3-0 reposicionando a camada muscular e subcutânea e sutura contínua simples em pele com Nylon 5-0.

Figura 3: A: Transoperatório. Acesso submandibular por planos.

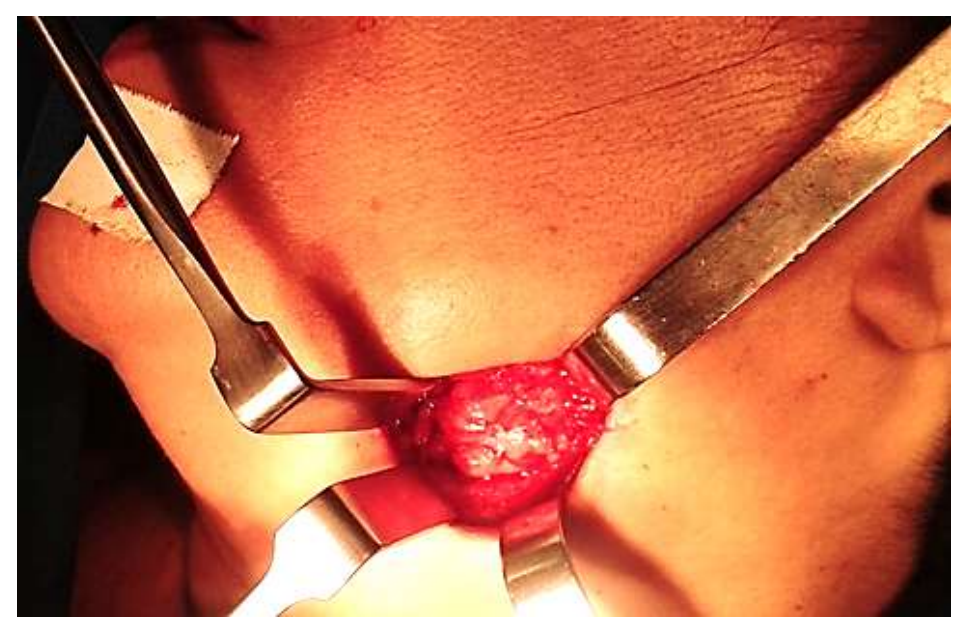

Fonte: Autores. 
Figura 3: B: Transoperatório. Identificação e exérese da lesão e glândula submandibular.

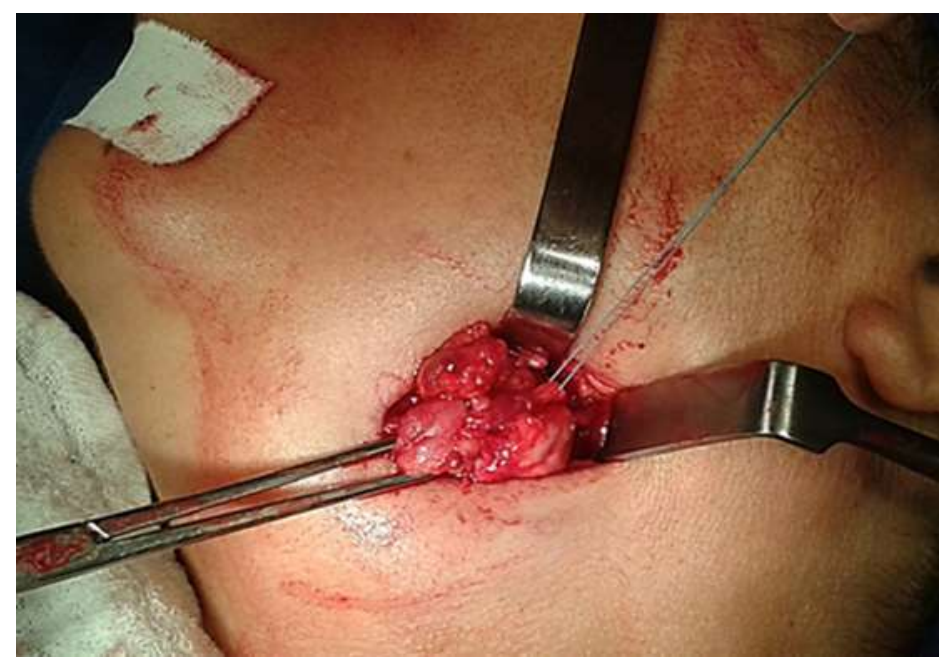

Fonte: Autores

Figura 3: C: Sialólito (embaixo) e glândula submandibular (em cima).

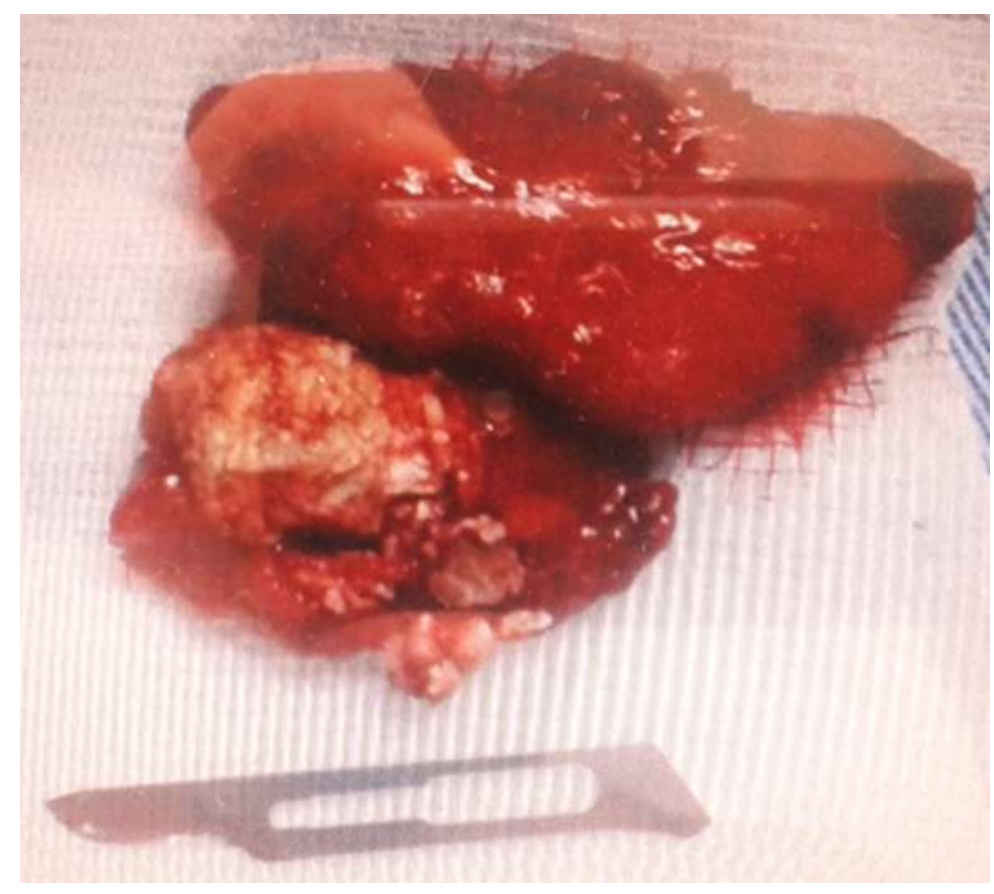

Fonte: Autores.

Na sala de recuperação anestésica, após paciente estar bem acordada, foi realizada avaliação da função motora do marginal mandibular, sem apresentar déficit (Figura 4). Paciente permaneceu 01 dia internada e no momento da alta hospitalar foi prescrito antibiótico (Amoxicilina 500mg, ingerir 01 cápsula de 8/8hs durante 07 dias); anti-inflamatório (Ibuprofeno 600mg, ingerir 01 comprimido de 8/8hs durante 05 dias) e analgésico (dipirona 500mg, ingerir 01 comprimido de 6/6hs por 02 dias). Passadas as orientações pós-operatórias. No exame de imagem pós-operatório de controle não foi constatado nenhum fragmento remanescente do sialólito. Bem como, não foram observadas complicações pós-operatórias como infecção, deiscência da sutura, déficit motor temporário ou permanente. A paciente está em acompanhamento pós-cirúrgico de 05 anos com adequada recuperação e restabelecimento estético funcional (Figura 5). 
Figura 4: Avaliação nervosa motora no pós-operatório.

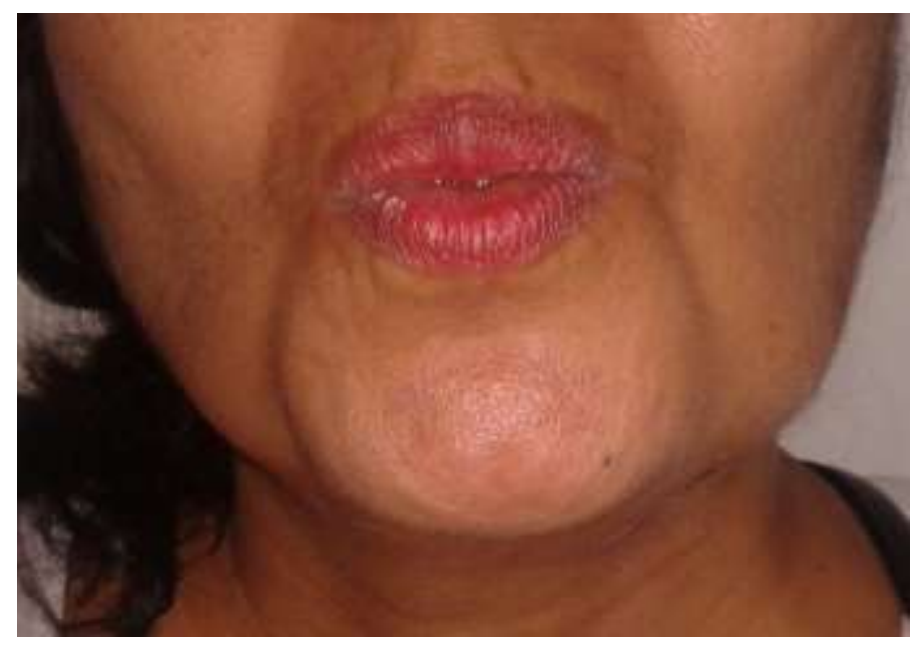

Fonte: Autores.

Figura 5: Avaliação no pós-operatório, não sendo observada nenhuma complicação motora.

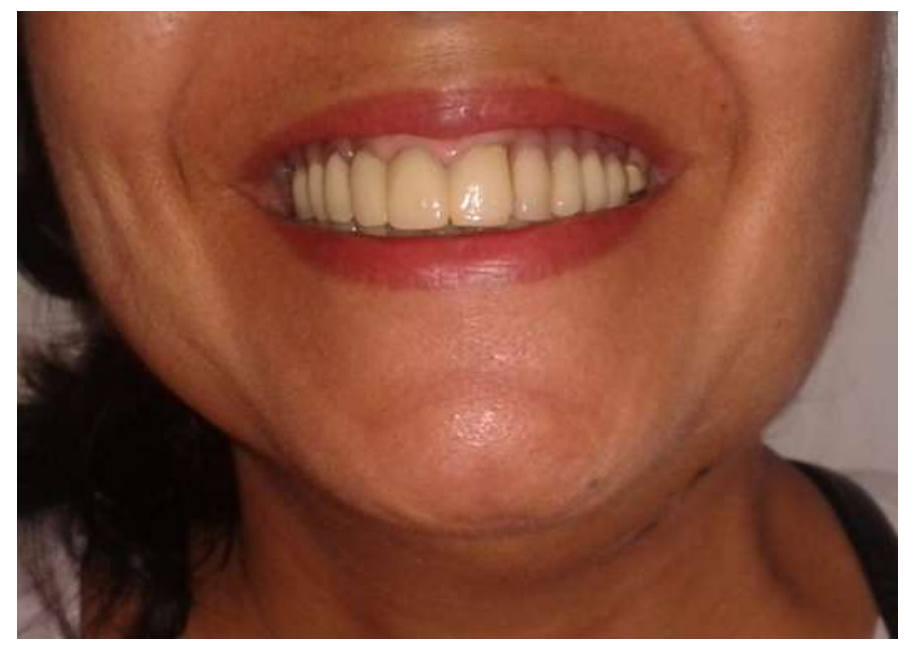

Fonte: Autores.

\section{Discussão}

A anatomo-fisiologia contribui para predileção na glândula submandibular, pois o ducto de Wharton apresenta um extenso canal tortuoso, a saliva secretada é mucosa, com $\mathrm{Ph}$ alcalino. Na topografia existe uma "área da vírgula", que é um local onde o ducto apresenta uma curvatura brusca, tudo isso explicaria a maior ocorrência. Quanto ao gênero, a literatura cita frequência masculina duas vezes maior que no feminino. (Miloro et al., 2016) Segundo Lima et al., (2013) e Oliveira et al., (2019), afirmam também predileção em homens, embora Neville et al., (2009) não trazem essa preferência por gênero algum. Nosso caso clínico, o sexo acometido foi oposto à predileção descrita pela maioria dos autores.

Kawata et al. (2006) e Silva et al., (2018) trazem como principal queixa, episódios álgicos e aumento de volume, devido a obstrução do ducto pelo cálculo. E que os sintomas dolorosos variam conforme o grau de obstrução. Araújo et al. (2011) e Fialho et al (2018), falam que impedindo o fluxo salivar, há risco de inflamação e infecção. Neste caso apresentado, apesar do cálculo ser de tamanho incomum, chegando aproximadamente $3 \mathrm{~cm}$ de diâmetro, a paciente encontrava-se com queixa álgica de pouca intensidade, sem sinais flogísticos.

Segundo Miloro et al., (2016), para o diagnóstico, além de um exame clínico minucioso, a sialografia seria o padrão 
ouro, no entanto Kignel et al., (2013) trazem que esse é um exame invasivo e doloroso, necessitando utilizar um meio de contraste no interior do ducto, além de anestesia local. Em 2014, Kraaij et al., Falam que a TCFC possibilita uma visualização nos três planos dimensionais, sendo essencial para diagnóstico não invasivo e para o planejamento terapêutico, o que faz desta uma excelente escolha. $\mathrm{O}$ exame complementar deste caso foi, inicialmente, a panorâmica para detectar o tipo de lesão, pois trata-se de um exame convencional, de boa visualização, embasado na literatura, sendo possível o diagnóstico de sialólitos maiores na glândula submandibular. Após detectar a lesão radiopaca, solicitamos uma tomografia computadorizada para avaliação em 3D, possibilitando planejar com precisão o tratamento.

A abordagem depende do tamanho e comprometimento do cálculo na glândula. Caso sialólito esteja na porção anterior, de tamanho pequeno, pode estimular a secreção salivar com sialogogos, dilatação do ducto e ordenha. Cálculo pequeno na porção posterior pode realizar incisão no local exato da massa, e posterior recanalização (Kawata et al., 2006). De acordo com Goes et al., (2013), sialólitos gigantes são mais comuns em mulheres e quando estão intraglandulares são tratados com a extirpação da glândula, com acesso intraoral, sendo essa uma gestão padrão. Miloro et al., (2016) discordam dessa posição e caso haja acesso limitado, pode sim ser necessário acesso extrabucal para a remoção da lesão e da glândula associados. Landgraf et al., (2006), afirmam que a sialadenectomia submandibular se faz necessária em cálculo gigante que se encontre intraglandular. Harmoniosamente com a literatura, tratando-se de lesão gigante no interior da glândula, optamos pela exérese do sialólito e da glândula, com acesso extraoral, já que concerne lesão gigante de dificultoso acesso intraoral.

Lima et al., (2013) falam sobre os tratamentos, que estes devem ser bem indicados, para evitar complicações póscirúrgica, como fibrose na área do ducto, fístula salivar, paralisia do nervo facial e deiscência da sutura. Araújo et al., (2011) completam trazendo relatos de que, quando acesso é extraoral, assim como neste relato de caso, a cicatriz facial e diminuição do fluxo salivar são as principais complicações. No caso aqui descrito, foi formada cicatriz facial estética, em área de difícil visualização em posição ergonômica, sem deiscência na sutura, nem déficit motor. A diminuição do fluxo salivar não foi considerável, estando a paciente em ótimo acompanhamento pós-operatório.

Hoje, com avanço tecnológico, existem outras modalidades terapêuticas, como sialoendoscopia, fragmentação por laser, porém são alternativas que exigem bastante experiência para que possam ser as melhores escolhas (Miloro et al., 2016). A exérese do sialólito e da glândula foi o tratamento escolhido para o caso citado acima, sendo baseado na literatura descrita, considerando o comprometimento glandular e difícil acesso, tratando-se de uma lesão gigante intraglandular.

\section{Considerações Finais}

Devido às variações existentes associadas às lesões orais, os sialólitos gigantes devem ser diagnosticados de forma precisa, através da anamnese minuciosa, exame extra e intraoral associados ao exame de imagem. O planejamento para a realização do tratamento deve ser de modo individualizado, optando sempre por tratamentos mais conservadores possíveis, tendo em vista as características da extensão e da localização do sialólito que, por sua vez, possuem predileção pela glândula submandibular.

\section{Referências}

Alves, N S., Soares, G. G., Azevedo, R. S., \& Camisasca, D. R. (2014). Sialolito de grandes dimensões no ducto da glândula submandibular. Rev. Assoc. Paul. Cir. Dent, 68(1), 49-53.

Araujo, F. A. C., et al. (2011). Tratamento cirúrgico de sialólito em glândula submandibular - relato de caso. Rev. cir. traumatol. buco-maxilo-fac. [online]. 11(4), 13-18.

Azenha, M. R., Brentegani, L. G., Silva, M. S., Rizoli, F. A., Lacerda, S. A., \& Filho, O. M. (2013). Sialolito de grandes proporções localizado no ducto da glândula submandibular: diagnóstico e tratamento cirúrgico. Odontol. Clín.-Cient. (Online), 12(1), 87-89.

Cardoso, A. M. R., \& Hoston, O. (2012). Sialolito Gigante em Ducto de Warton:Relato de Caso Clínico. Rev. bras. ciênc. Saúde, 16(03), 459-462. 
Research, Society and Development, v. 10, n. 15, e541101523526, 2021

(CC BY 4.0) | ISSN 2525-3409 | DOI: http://dx.doi.org/10.33448/rsd-v10i15.23526

Dall'Magro, A. K., Alves, C. C., Lauxen, J., Santos, R. Valcanaia, T. C., \& Dall’Magro, E. (2014). Sialólito de glândula salivar submandibular: relato de caso. RFO UPF, 19(3), 343-347.

Fialho, P. V., Dantas, B. P. L. S. S., Fernandes, A. C. F., Silva, D.T., \& Queiroz, C. S. (2018). Sialolito Em Parênquima De Glândula Submandibular: Relato De Caso. Rev. Odontol. Araçatuba (Impr.), 39(1), 9-14.

Goes, P. E. M., Lima, V. N., Carvalho, F. S. R., Queiroz, S. B. F., \& Camargo, I. B. (2013). Sialolito gigante em ducto de Wharton: um caso distinto e revisão da literatura. Rev. cir. traumatol. buco-maxilo-fac, 13(4), 81-88.

Kawata, L. T., et al. (2006) Sialolitíase em ducto de glândula submandibular: relato de dois casos clínicos. Revista Odontológica de Araçatuba, 27(1), 14-18.

Kignel, S. et al. (2013). Estomatologia - Bases do diagnóstico para o clínico geral. (2ª. Ed.), 2013.

Kraaij, S., et al. (2014). Salivary stones: symptoms, aetiology, biochemical composition and treatment. Br Dent J, 217(11), 637-638.

Landgraf, H., et al. (2006). Extenso sialolito no ducto da glândula submandibular: relato de caso. Rev. Cir. Traumatol. Buco-maxilo-fac., 6(2), 29-34.

Leite, R. B., et al. (2018). Sialólito Gigante em Ducto de Wharton: um caso incomum. Rev. Cir. Traumatol. Buco-Maxilo-Fac., 18(1), 34-38.

Lima, A. N., Milani, B. A., Massaine, L. F. Souza, A. M. M., \& Jorge, W. A. (2013). Sialolitíase em glândula submandibular: relato de caso clínico. Rev. Cir. Traumatol. Buco-Maxilo-fac., 13(1), 23-28.

Manzi, F. R., Silva, A. I. V., Dias, F. G., \& Ferreira, E. F. (2010). Sialolito na glândula submandibular: relato de caso clínico / Sialolith of the submandibular salivary gland: case report. ROBRAC, 19(50), 270-274.

Miloro, M., et al. (2016) Princípios de cirurgia bucomaxilofacial de Peterson. (3ª ed.): Santos.

Neville, B. W., et al. (2009) Patologia oral e maxilofacial. (3ª. ed.): Elsevier.

Oliveira, M. M. M., Acioly, R. F., Souza, Dennis D. S., Silva, B. A., \& Carvalho, D. C. (2019). Sialolito de grandes dimensões em ducto de glândula submandibular: relato de caso. Rev. cir. traumatol. buco-maxilo-fac, 19(2), 33-36.

Pereira, A. S., et al. (2018). Metodologia da pesquisa científica. [e-book]. Santa Maria. Ed. UAB/NTE/UFSM.

Pretto, J. L. B., Machado, R. A., Silveira, R. L., Borges, H. O. L., \& Pagnoncelli, R. M. (2007). Sialolito em glândula submandibular - relato de caso. RFO $U P F$, 12(3), 61-64.

Silva, F. B. M., Nogueira, B. O., Alves, A. T. N. N., Uzeda, M. J. P. G., \& Resende, R. F. B. (2018). Diagnóstico e tratamento de Sialolitíase: Relato de Caso. Rev. cir. traumatol. buco-maxilo-fac, 18(2), 25-29. 